\title{
BMJ Open Understanding the investigators: a qualitative study investigating the barriers and enablers to the implementation of local investigator-initiated clinical trials in Ethiopia
}

Samuel R P Franzen, ${ }^{1}$ Clare Chandler, ${ }^{2}$ Fikre Enquselassie, ${ }^{3}$ Sisira Siribaddana, ${ }^{4}$ Julius Atashili, ${ }^{5}$ Brian Angus, ${ }^{6}$ Trudie Lang $^{1}$

To cite: Franzen SRP, Chandler C, Enquselassie F, et al. Understanding the investigators: a qualitative study investigating the barriers and enablers to the implementation of local investigator-initiated clinical trials in Ethiopia. BMJ Open 2013;3:e003616. doi:10.1136/bmjopen-2013003616

- Prepublication history for this paper is available online. To view these files please visit the journal online (http://dx.doi.org/10.1136/ bmjopen-2013-003616).

Received 18 July 2013 Accepted 24 October 2013

CrossMark

For numbered affiliations see end of article.

Correspondence to Samuel R P Franzen; sam@globalhealthtrials.org

\section{ABSTRACT}

Objectives: Clinical trials provide 'gold standard' evidence for policy, but insufficient locally relevant trials are conducted in low-income and middle-income countries. Local investigator-initiated trials could generate highly relevant data for national governments, but information is lacking on how to facilitate them.

We aimed to identify barriers and enablers to investigator-initiated trials in Ethiopia to inform and direct capacity strengthening initiatives.

Design: Exploratory, qualitative study comprising of in-depth interviews $(n=7)$ and focus group discussions $(n=3)$.

Setting: Fieldwork took place in Ethiopia during March 2011.

Participants: Local health researchers with previous experiences of clinical trials or stakeholders with an interest in trials were recruited through snowball sampling $(n=20)$.

Outcome measures: Detailed discussion notes were analysed using thematic coding analysis and key themes were identified.

Results: All participants perceived investigatorinitiated trials as important for generating local evidence. System and organisational barriers included: limited funding allocation, weak regulatory and administrative systems, few learning opportunities, limited human and material capacity and poor incentives for conducting research. Operational hurdles were symptomatic of these barriers. Lack of awareness, confidence and motivation to undertake trials were important individual barriers. Training, knowledge sharing and experience exchange were key enablers to trial conduct and collaboration was unanimously regarded as important for improving capacity.

Conclusions: Barriers to trial conduct were found at individual, operational, organisational and system levels. These findings indicate that to increase locally led trial conduct in Ethiopia, system wide changes are needed to create a more receptive and enabling research environment. Crucially, the creation of

\section{Strengths and limitations of this study}

- To our knowledge this is the first empirical research study exploring investigator-initiated trial implementation in low-income and middle-income countries.

- Gives a compelling voice to local investigators who are often unheard in this Northern dominated discourse.

- As a formative study, the sample size was small, the sampling purposive and the findings may be context specific.

research networks between potential trial groups could provide much needed practical collaborative support through sharing of financial and project management burdens, knowledge and resources. These findings could have important implications for capacity-strengthening initiatives but further research is needed before the results can be generalised more widely.

\section{INTRODUCTION}

Many development organisations argue that clinical research in low and middle income countries (LMIC) is essential for improving public health and development. ${ }^{1}$ In LMICs clinical research capacity remains insufficient. This perpetuates the '10/90 gap', where only $10 \%$ of global health research expenditure is allocated to diseases that primarily affect $90 \%$ of the world's population. This leads to a lack of evidence of the world's most burdensome diseases. ${ }^{2}$ Evidence from Northern nations is often not relevant to LMICs, ${ }^{3}$ and its adoption into 
clinical practice can be slow and treated with caution. ${ }^{14}$ Increasing the number of clinical trials conducted in LMICs would help generate local evidence, ${ }^{4}$ which may be more likely to rapidly influence policy and practice. ${ }^{5}$

Most clinical trials conducted in LMICs are run by foreign research organisations with their own agendas. Recently there have been calls from within LMICs for more ownership over priority setting, ${ }^{6}$ greater engagement with local research communities ${ }^{7}$ and research conducted in line with national health strategies. ${ }^{8}$ Pragmatic, locally initiated disease management studies could significantly improve public health. ${ }^{4}$ Despite being simple and cost-effective, they are often ignored by international trial groups, ${ }^{9}$ and are rarely independently undertaken in LMICs. By 'pragmatic' and 'simple' we are referring to studies that are designed to test effectiveness, have few endpoints and broad eligibility criteria, thereby increasing external validity. ${ }^{10} 11$ Meanwhile, there is increasing expectation that LMICs should take more responsibility for their research activities. ${ }^{12}$ Increasing the number of local investigator-initiated trials (IITs) could be an answer to these issues. Several advantages of IITs over foreign-initiated trials (FITs) have been put forward (box 1).

The establishment of African-owned research centres capable of running their own clinical trials has been identified as an international priority, ${ }^{17}$ and there are ever increasing numbers of clinical trials being conducted in LMICs. ${ }^{4}$ However, few of these trials are locally initiated, ${ }^{18}$ and globally, trials are becoming harder to implement. ${ }^{4}$ Many research bodies have increased efforts to support IITs in Europe, ${ }^{18}{ }^{19}$ but within LMICs capacity building mostly focuses on developing sites to run international trials. Capacity building to support independent locally led trials is likely to require a different approach. However, little is known about the best way to develop capacity and facilitate their conduct. This formative study investigates the issues facing local trial investigators in Ethiopia, and was designed to begin addressing this knowledge gap by determining the barriers and enablers to trial conduct.

Box 1 Advantages of investigator-initiated trials over foreign-initiated trials

More applicable to local populations due to building on local healthcare knowledge ${ }^{13}$

- More demand-led and responsive to a country's needs because they are driven by a national agenda ${ }^{9}$

- More likely to influence policy ${ }^{13}$ and sustainably link research to action ${ }^{14}$

- Often simple studies that address important topics such as disease management ${ }^{4} 9$

- Involve local staff at all levels and stages of trial conduct, ${ }^{15}$ so there is more opportunity for 'learning by doing' and skill development ${ }^{16}$

\section{METHODS}

We conducted a qualitative study using in-depth interviews (IDIs) and focus group discussions (FGDs). Research exercises took place in Addis Ababa and Gondar, Ethiopia, in March and April 2011. Ethiopia was selected to represent a country in Sub-Saharan Africa that conducts a modest number of clinical trials while having sufficient trial experience to contribute to this study. Ethiopia had 39 trials registered at the time of fieldwork; a breakdown of these by intervention and sponsor is shown in figure 1 . The majority of drug trials investigated the use of approved drugs to optimise treatment.

This study seeks to understand the perspectives and experiences of current and potential trial investigators and staff. Owing to the paucity of previous work on this topic, we did not prospectively adopt a specific theoretical framework. However, research questions were influenced by the fields of organisational change and development. FGDs and IDIs were semistructured and explored the following themes: the clinical research environment in Ethiopia, barriers and enablers to trial conduct, access to appropriate skills and knowledge, current support for clinical trials and recommendations for change. Questions were tailored to participant experience and emerging themes.

Participants were identified first through trial registration searches and subsequently by snowball sampling from these individuals. Health researchers with previous experiences of clinical trials or stakeholders with an interest in trials were selected. Of all the participants approached, none refused to take part. Interviews and discussions were conducted in English, and explored key points until no new information emerged. In preliminary meetings, participants said they would speak more openly if discussions were not audio recorded. This was because they would be uncomfortable criticising partners or regulatory bodies while being recorded. One participant explained that this worry was a result of the legacy left by previous authoritarian regimes. Detailed notes were taken with quotes noted as near verbatim as possible, detailing identification numbers.

This study was approved by the University of Oxford Tropical Research Ethics Committee. Verbal informed consent was obtained from all participants and review of discussion contributions and written confirmatory permission was obtained for all participants who could be contacted $(15 / 20)$. No quotes are included from those who could not be contacted.

Notes were analysed by thematic coding analysis using Nvivo qualitative data analysis package (QSR International Pty Ltd. V.9, 2011) to help organise the data. Data were coded inductively according to its semantic content. ${ }^{20}$ Using relationship and modelling functions, a mechanistic model of factors influential to clinical trial conduct was developed through the piecing together of complementary segments of data contributed from different participants. Coding was completed 
Figure 1 Number of clinical trials in Ethiopia by intervention type and sponsor, as registered on the WHO International Clinical Trials Registry Platform in the same period as data collection (30 April 2011). The observational study was registered as a clinical trial but was a cohort study with a nested cross-sectional design.

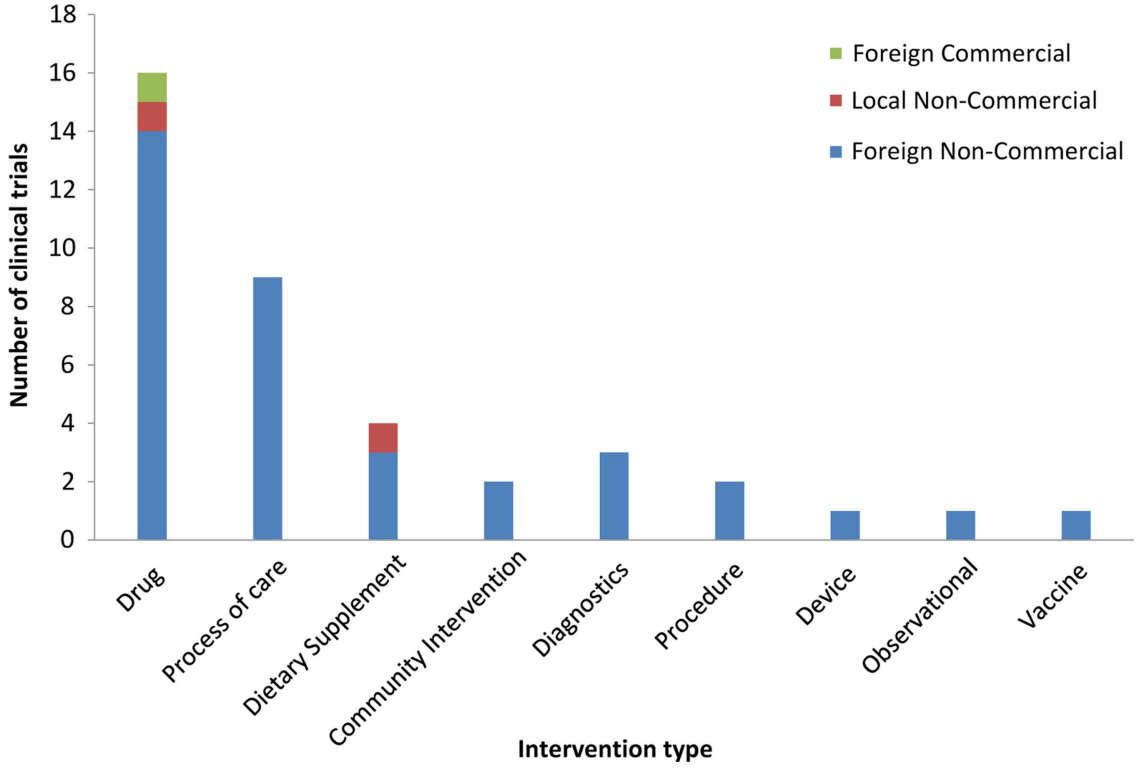

by $\mathrm{SF}$ with consultation and agreement from other authors (TL, CC and BA). Findings were reviewed and commented on by all authors.

\section{RESULTS}

\section{Study population}

We conducted two FGDs and six interviews in Addis Ababa and one FGD and one interview in Gondar. A total of 20 researchers participated; seven were based at a research centre, one at an NGO, eight at a hospital and four at a university. Participants had varied job roles. Those currently working on a clinical trial included: senior investigators $(n=2)$, trial managers and coordinators $(n=5)$, laboratory personnel $(n=5)$ and research nurses $(n=2)$. We also recruited six medical

\begin{tabular}{|l|l|}
\hline \multicolumn{1}{|c|}{$\begin{array}{c}\text { Medical professional experience } \\
\text { domains }\end{array}$} & $\begin{array}{c}\text { Number of participants } \\
\text { with experience (one } \\
\text { participant may have multiple } \\
\text { experience domains) }\end{array}$ \\
\hline Clinical trial experiences & \\
\hline No clinical trial experience & 3 \\
\hline Foreign initiated trials & 15 \\
\hline Local investigator initiated trials & 3 \\
\hline Other experiences & \\
\hline Academia & 8 \\
\hline Clinical Practitioner & 9 \\
\hline Public health practitioner & 3 \\
\hline University research leadership & 5 \\
\hline Ethics board member & 3 \\
\hline Government worker & 1 \\
\hline
\end{tabular}

Figure 2 Participant experience of different medical professional experience domains. Experience domains are not mutually exclusive and one participant can have multiple experience domains. researchers not currently working on a clinical trial, three of whom had previous trial experience and three that did not. The participants had experience in a diverse range of medical professional experience domains (figure 2).

\section{A role for investigator-initiated trials}

All participants reported that too few clinical trials are conducted in Ethiopia, and felt this limited the ability for guidelines to be based on local evidence. Most treatment strategies were based on international guidelines, which many participants thought could be inappropriate. Participants proposed that locally-led trials would be useful for filling this evidence gap and that the conduct of simple design studies was independently achievable. Many researchers would like to lead their own studies and had important questions, but were often unsure of how to go about doing this, as described by this senior clinician:

We don't have the evidence to change local practices but we definitely know some written guidelines don't work. There are a number of unanswered questions for trials but we don't know how to do them. We need clinical research [in disease areas] that has a different effect in Ethiopia, for example HIV and TB. These diseases are similar as to other places but we have had little success [controlling them] here. So why? Where are the mistakes? These sorts of investigations are easy, they would support awareness and fill gaps. FGD -3 PPT-1

Compared to foreign-led studies, local trials were perceived to be more likely to address evidence gaps, be more useful for developing treatment guidelines and more sensitive to community issues. One junior clinician described this through his experiences:

Investigator-initiated trials increase evidence, particularly locally relevant evidence. For instance the Leishmania 
strain in Gondar seems to be a bit different to the other strains because the drug treatment is working better in the other areas. This will prevent mistakes in clinical care. INT-6 PPT-1

\section{Human and material capacity}

While a general lack of materials, infrastructure and laboratory facilities were thought to reduce the number and scope of trials, most participants felt that human resources were the critical factor. Respondents stated that there were too few investigators with the technical expertise to initiate a trial. There was also a shortage of skilled research staff, with one investigator stating that if one or two staff left, their trial could not continue (FGD -3 PPT-1 senior clinician and trial investigator). This lack of expertise and research skills was blamed on minimal research focus in clinical education, few opportunities to gain experience and few local experts who could share their knowledge.

From my undergraduate experience, I was trained to be a clinician and not a researcher; this is from a curriculum point of view. Few clinicians use clinical trials as they are involved in primary care and not research. They have no spare time to think about research... We need the opportunity to have a simple role and experience to get more people to do more trials. As more people get involved in simple research and trials, more research will be done. INT -6 PPT-1, trial clinician

Individuals skilled in trials were often too busy with regular duties to be able to conduct research. Most senior trial staff were clinicians and while release from routine duties could be negotiated, they complained that healthcare tasks still had to be prioritised. Academics had allocated time for research but this was regularly cited as insufficient. The limited manpower allocation to research and few opportunities to gain experience resulted in a negative feedback loop, as explained by this trial clinician:

\footnotetext{
The scope of activities is narrowed by the time and economic constraints and the fact few individuals can be involved. Because the scope is narrowed this results in a cycle of fewer people being involved, which in turn results in less motivation, fewer trials and less exposure and realising the way of thinking and less achievement. Then less people are involved and there are fewer trials. INT -6 PPT-1
}

\section{Regulatory and other administrative bottlenecks}

Respondents reported that complex and strict government regulations made it very difficult to investigate novel interventions and recruit vulnerable populations. Regulatory and ethical review times also introduced delays and it was not uncommon for grants to expire before all approvals were in place. A trial principal investigator (PI) explains this further:
Regulatory authorities have no clear guidelines so it is problematic getting approvals. They are also not experienced enough and so are overcautious and they cannot decide on interpretations. FGD-1 PPT-1, Trial PI

Ethics committee members admitted that limited resources, knowledge gaps and membership shortages slowed review times, but also pointed out that poor quality applications meant resubmission was regularly required. They emphasised that clarification of regulations and developing review capacity were essential to facilitate trial implementation and that more training in research ethics was needed. One participant explained that The Ethiopian Bioethics Initiative is already working towards this. Funded by the European and Developing Countries Clinical Trial Partnership, The Ethiopian Bioethics Initiative helps research sites to form Institutional Review Boards (IRBs) and train the committee members on basic principles of ethical clearance. Under this grant they have established and trained 11 IRBs.

University and government administration systems were unanimously regarded as overly complicated and blamed for many operational delays. To cope with this, many investigators said they required an administrative assistant but could not afford one.

The university finance department is a bottleneck. You really need an admin assistant to help with this. Most doctors do not want to go through the pain of organising and administrating all this. Also if you do not report your annual budget on time you may be penalised and have your salary suspended. The clinical trial financing really increases the amount of work you must do for your budget reporting. If I got a good grant I would hire an admin or research student to manage these issues. INT3 PPT-1, clinician

\section{Operational hurdles}

During one in-depth interview, a process mapping exercise was used as a template for discussion; the participant draws a detailed flow diagram of the steps and tasks involved in conducting their clinical trial, noting problems, successes and changes to be made in hindsight. Box 2 describes the experience of one PI, providing an example case of operational hurdles and the importance of advanced planning.

The majority of serious operational difficulties occurred during the start-up stage of trial conduct. Once intervention delivery began, there were few major challenges. Participants attributed most operational hurdles to wider issues. The main operational hurdles and their causes are summarised in table 1. Operational enablers included: keeping trial designs simple, only investigating approved interventions and non-vulnerable populations to prevent regulatory delays, and rapid recruitment of participants due to large patient pools that were usually prepared to give consent. 
Box 2 Experience of a local principal investigator (PI) on a foreign non-government organisation-led non-commercial study

Registering the clinical trial caused considerable delays because task allocation was overlooked. Ethical approval for this relatively complex study took 12 months due to a cycle of resubmission. Ordering to delivery of supplies routinely took 3-6 months. Data entry and analysis were delayed because the data management system had not been considered early enough and training could only be obtained in Europe. Some laboratory tests were outsourced to a local private laboratory because staff lacked the training and equipment, while other assays were complicated by lack of normal ranges for local populations. In hindsight the PI would have taken more care with planning and preparation and made roles and responsibilities clear from the outset.

\section{Initiating an idea: awareness, confidence and motivation}

Participants reported that limited awareness of trial research among their colleagues was a common reason for trials not being attempted. This was because potential

Table 1 Participant reported operational hurdles and their causes

Operational hurdles
$\begin{aligned} & \text { 1. Difficulty writing } \\ & \text { proposals and gaining } \\ & \text { funding }\end{aligned}$

2. Slow regulatory and ethical approvals

\section{Reported causes}

- Little local funding, competitive international funding

- Technical ability and confidence lacking

- Unsure of funding process; investigators do not complete application

- Lack of training

- Complex and unclear guidelines

- Limited ethical review capacity

- Poor-quality submissions

3. Problems with trial management

- Lack of experience and poor planning

4. Burdensome administration and difficulty purchasing supplies

- Complex and slow systems

- No administrative support

5. Problems with setting up and running laboratory tests investigators were not exposed to the methodology or had not considered doing them, as explained by this PI:

People do not have the vision that clinical trials will improve patient care because they do not see it in their daily lives. Clinical trials are important but essentially people do not see them enough to think of them. INT-4 PPT-1, senior clinician

This was attributed to omissions in medical student curricula, limited access to literature, little trial research training and few trial opportunities. If individuals had considered conducting a trial, many participants said that most researchers were not confident to initiate one themselves because they lacked the knowledge and skills. Even investigators who had considerable experience on foreign-led studies said they did not feel ready to lead their own. The expectation of operational difficulties and few examples of role models successfully conducting trials created a 'phobia' of trial research, with people believing them to be almost impossible. This 'phobia' and lack of awareness was seen as a key barrier to trial conduct, as explained by this junior trial investigator:

We need to develop and support a research culture by capacity building to develop skills and resources, not big capacity building like an operating room, but small scale like small grants for beginner researchers to do research and get practice-this would take away the phobia of writing proposals and publications. When the phobia has gone there will be floods of research. We need to open our eyes and see what can be done. For instance people don't know how to write a proposal. In people's academic studies this sort of stuff is not given priority. Even small research will be an eye opener and the phobia will be gone. FGD -3 PPT - 2, clinician and junior investigator

Even when potential investigators felt ready to conduct a trial, many participants said that the motivation for undertaking them was insufficient and this discouraged their colleagues from attempting them. Participants were encouraged by altruistic incentives such as community health improvement and organisational development but personal career incentives were weak. A lack of research career options discouraged students from entering into research after studies or caused them to migrate for work, as this junior academic explained:

We need to make sure people get jobs and an established career in research to get them to stay in their home country or to come back to their home country after training or education abroad. INT - 2 PPT-1

Researchers also reported little recognition for research and that promotion could be achieved without doing research. Additionally, strong salary and workload disincentives were cited, as summarised by this IDI participant: 
The problem is that this [clinical trial research] will take lots of your time and while it is possible to reduce your clinic hours, this means you would lose money. The country does not pay you even though it is for public benefit. There is no incentive, in short". INT-4 PPT -1, senior clinician

\section{The key enablers: training, knowledge sharing and experience exchange}

Equal to their value for building technical competence, many participants saw trial training, knowledge sharing and experience exchange as key enablers for increasing awareness, confidence and motivation. Training was viewed as important for awareness and encouraging staff to consider their workplace challenges in a more enquiring light. Knowledge sharing boosted a researcher's confidence that trials were achievable and experience exchange was important for raising professional standards and dispelling what one respondent termed 'pseudo-confidence' (INT-4 PPT-1-Clinician and trial $\mathrm{PI}$ ); meaning to continue working in a suboptimum way because knowledge of more rigorous methods was lacking. Many participants emphasised that all these enablers would be more effective if grounded in local examples and context, such as knowledge sharing with individuals whose settings were similar to the researcher's own. Learning activities were also highly motivational because they were prized for both personal and professional development. Given the scarcity of trial opportunities in Ethiopia, some participants suggested that national or international experience exchange programmes would be useful. One FGD participant explained her trial team's experiences:

What we really want is for a south-south collaboration like Kenya or Uganda to do exchange placements for our junior staff. This would show people in resource-limited settings it is possible to do trials and would motivate people much more than website or e-learning...She [referring to research nurse] has been to Kenya and Switzerland and this has helped her to see how clinical trials are done and what is good and bad and see the possibilities. FGD-3 PPT-1, senior clinician and trial investigator

\section{The importance of collaboration}

While some respondents had negative experiences with foreign led-studies or said they would prefer to be less dependent on foreign groups, most participants were very positive about international collaborations, assuming intellectual independence could be protected. Technical expertise and infrastructure strengthening provided through collaborations were consistently proposed as a solution to the limited human and material resources. Particularly, collaborative grant applications had been very helpful for securing funding by increasing the quality and credibility of applications. Local and international collaboration was also seen as a key way to access and promote training, knowledge sharing and experience exchange. This senior academic summarises the general opinion:

The priority is addressing local concerns like field-based optimisation. Weight should be given to locally initiated ideas. However, you should then ask for international assistance and collaboration. The investigator-initiated trial is all about the idea and not about the operation. You do not have to re-invent the wheel; you should make the most of global knowledge and skills. Everyone should chip in with their appropriate competence and expertise. This way the work will be faster and more efficient and local researchers will have access to technologies. INT-1 PPT-1

However, junior participants said they lacked the contacts and knowledge to develop partnerships and even established researchers often felt intellectually isolated from the East African and wider research community. This was believed to hamper innovation and cause repetition of ideas.

\section{DISCUSSION}

This study highlights that Ethiopian investigators think that investigator-initiated trials would generate highly useful and applicable data, supporting the call for more local evidence generation in LMICs. The challenge is implementing and successfully conducting a locally led study. We have identified barriers to the implementation of investigator-initiated trials in Ethiopia at all levels of the research system. Exploring through the perspective of local investigators has given a critical understanding of how these issues influence their ability to initiate trials. We have demonstrated the importance of training, knowledge sharing, experience exchange and collaboration, for breaking down barriers in somewhat unexpected ways and now consider, in light of this, how locally-led trials can be better supported.

\section{The research system}

Health research systems represent the coordinated activities of all stakeholders to produce health research and may operate at local, national, regional or global levels. The four main functions of health research systems are: stewardship, financing, resources and producing and using research. ${ }^{21}$ In Ethiopia, barriers and enablers to trial conduct have been identified at all levels of the national research system; system, organisational and individual. The main influential factors identified in this study have been summarised into a mechanistic model (figure 3). The following description is intended to illustrate the interconnected nature of the barriers to trial research and how deficiencies at one level can have cascading negative effects. System level barriers impact on all levels through often dysfunctional regulatory and administrative systems, insufficient funding allocation and limited ethical review capacity. Suffering from limited resources, the organisational level provides 


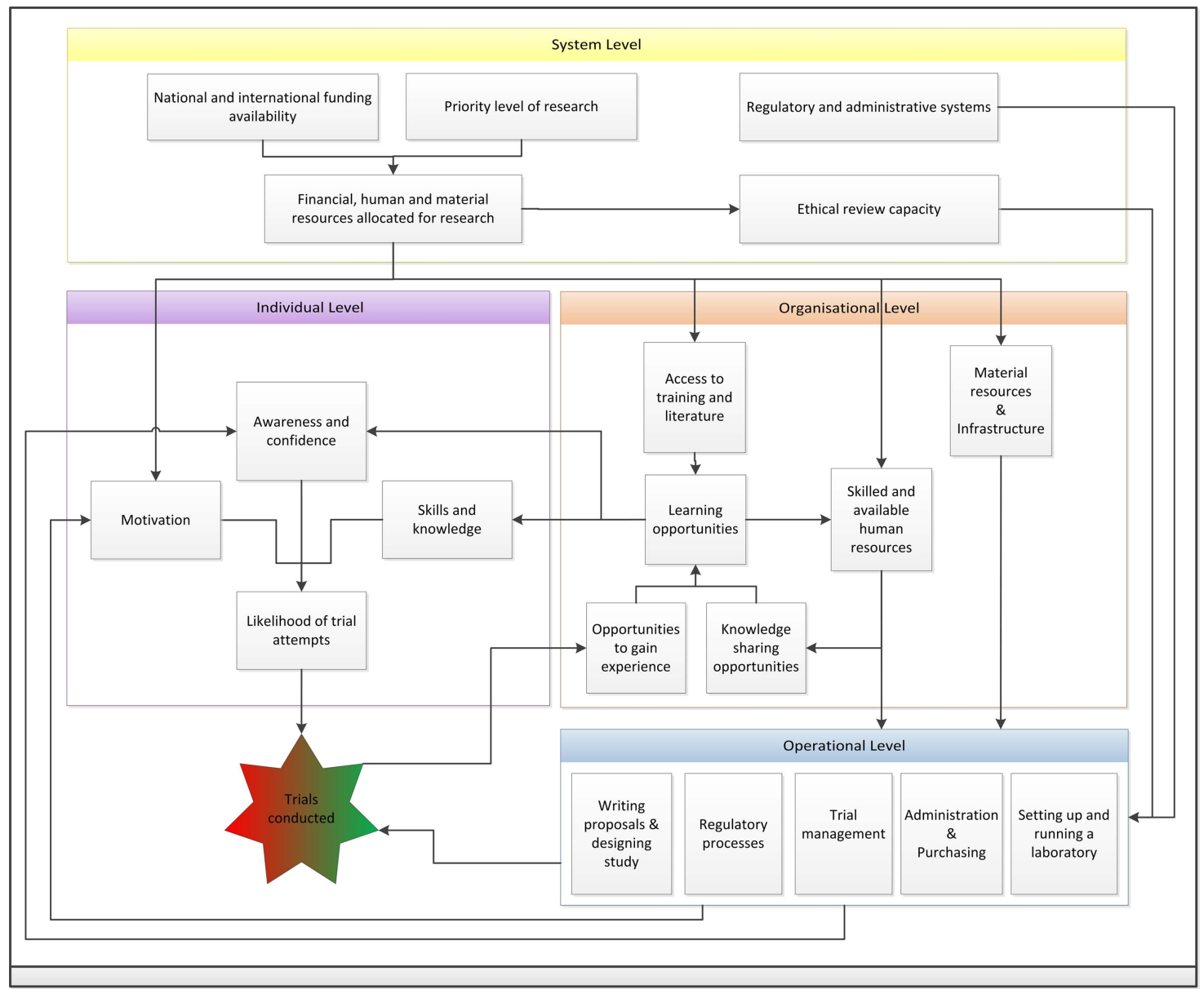

Figure 3 Mechanistic model of the factors influencing clinical trial conduct in Ethiopia.

limited learning opportunities, which negatively impacts on human resources. These deficiencies, combined with adverse regulatory and administrative systems, make operating clinical trials difficult. The combined effects of insufficient resources, limited learning opportunities and difficult operations result in a disabling research environment at an individual level. This reduces awareness of trials, limits competence and confidence and reduces motivation to undertake them. Few trials are attempted and this forms a negative feedback loop by reducing opportunities for experience.

A detailed review of the Ethiopian Health Research System also cites slow regulatory and ethical review, difficult administration systems, limited human resource allocation and few incentives as major impediments. ${ }^{22}$ However, it is important to emphasise that our description is not universal and individual examples of enabling practices and trial capacity exist. Furthermore, no information was available to us on the number of trial applications, rejections and turnaround times and although little local evidence-based practice was reported, this was not confirmed by the authorities and examples of local trials influencing policy are found in the literature. ${ }^{23}$ Nevertheless these results demonstrate the importance of taking a system-wide view to research development.
Operational problems are embedded in wider issues, and while certain strategies may help investigators cope with problems, their resolution is dependent on strengthening the capacity at all levels of the system. Fortunately in-country expertise exists in almost all major aspects of the health research system, which should greatly facilitate strengthening efforts. ${ }^{22}$

\section{Building a receptive research environment}

Although the capacity to conduct trials was limited, most researchers agreed that simple design studies could be carried out. However, local investigators have attempted few trials. A key problem was the disabling research environment at the individual level. Clinical trials are a relatively new phenomenon and are not yet embedded in the Ethiopian research culture. Therefore, their implementation can be viewed as a change within this culture. The Awareness, Desire, Knowledge, Ability and Reinforcement (ADKAR) model for organisational change management, suggests that for a change to happen at an individual level, ADKAR must be present. ${ }^{24}$ Trial awareness was limited and investigators did not have the vision that trials would improve patient care. Although most participants had the desire to lead a trial, they reported a general lack of knowledge and 
competence. For those that had the knowledge, many still felt unable to lead a trial, or were unwilling because of minimal motivational reinforcement.

This study, and others, ${ }^{25} 26$ suggests that increased learning opportunities and rewarding career paths are required to increase human resource capacity and retain skilled personnel. Increasing research components in taught courses, and providing training and small research grants for young researchers would increase awareness, desire and knowledge to conduct research. In Ethiopia, clinical academic staff have low salaries and are paid less than public sector physicians. ${ }^{27}$ This, combined with limited funding, high-teaching burdens, lowquality facilities and frustrations with bureaucratic and operational hurdles found in this and another study, ${ }^{27}$ all serve as strong disincentives to research. Providing protected time for research and recognising research within careers would help motivate investigators to undertake trials through positive reinforcement, but this alone may not be sufficient without adequate salaries to offset lost revenue from private practice and consultancy work.

Several participants expressed their inability to lead their own trial as a lack of confidence, or phobia and investigators who had worked on foreign-led studies still did not feel ready to lead their own trial. Local funding and material capacity constraints were often cited as the cause, but many researchers had not applied for international funding. Expectation of insurmountable barriers and lack of successful role models certainly reduce confidence. However, it is possible that the intellectual isolation identified in this and another study, ${ }^{27}$ and lack of a supportive research environment, could reduce initiative. We also propose that previous regimes that actively discouraged autonomy, could have left a legacy that reduced the ability of individuals to act as agents in change.

\section{Networking}

Collaboration was key to the provision of training, knowledge sharing and experience exchange and accessing technical expertise and infrastructure strengthening. Better networking within and between local and international research organisations could facilitate the provision of these enablers, decrease intellectual isolation and help develop a National Health Research System. ${ }^{22}$

Successful local sites are often busy, so exchange placements could be organised to allow more individuals to get involved. This could potentially increase trial staff numbers and reduce the impact of task shifting on clinic work. This may then reverse the negative feedback loop between insufficient human resources and few trials. Local research networks could support sustainable training models if a few experts become trainers of trainers and mentorship programmes linking experts to junior staff could provide inspirational support and guidance for isolated individuals. The need for administrative support in LMICs is common as many universities lack established research services. ${ }^{26}{ }^{28}$ Cooperation between departments to form research clusters would make hiring an administrative assistant affordable and could help share the cost and burden of purchasing supplies.

The creation of communities of practice could help to develop these relationships but proven strategies that foster their development are not clearly established. ${ }^{29}$ Networking opportunities such as workshops would be useful, but first, all stakeholders need to be identified, and travel, time and cost can be barriers. Also, the informal nature of partnership formation in this study meant that less established researchers did not have the contacts or knowledge to find partners. One solution could be to develop a local and international online networking facility, detailing research interests, expertise, resources and current projects.

\section{Prioritising research systems}

Capacity building attached to foreign-led studies typically focuses on training individuals in specific skills or providing one specific service or resource. While this may get a particular study completed, in Ethiopia, it does not appear to provide the package necessary to allow local investigators to conduct their own trials. This study demonstrates the diversity of factors influencing IIT implementation and shows that focusing on system wide improvements must be integral to any long-term research development plan for Ethiopia. For this to happen, we suggest that research must be prioritised, not just in terms of resource allocation but also the value placed on research. However, in a country with insufficient health workers to provide routine healthcare $^{27}$ and many other competing priorities, this may be difficult.

Despite this, such a value change could already be underway. The Ethiopian Science and Technology Agency devised strong implementation strategies to support research in $2006^{30}$ and built on them in $2012 .{ }^{31}$ The importance of research and developing research capacities is now central to the Ministry of Health strategy, and local trial data ${ }^{32}$ has influenced policy. ${ }^{33}$ Recommendations for fostering a research culture in the New Public Universities have been developed ${ }^{34}$ and the Jimma University in South West Ethiopia has been applauded for pioneering new innovative teaching methods, valuing research within institutional culture and integrating it in career progress. ${ }^{27} 35$ Meanwhile, The Ethiopian Bioethics Initiative is working hard to strengthen regulatory procedures and provides a successful example of developing ethical review capacity.

\section{Strengths and limitations}

As a formative study, the sample size and range of stakeholder roles was small, and the findings may be specific to the locations or dependent on contextual factors such as governance style. Although this limits the breadth of perspectives and generalisability of findings, the study sample 
accessed diverse experiences and used in-depth qualitative methods to uncover issues in a largely unexplored area, while giving a compelling voice to local investigators who are often unheard in this Northern dominated discourse. The inability to audio record the discussions may have had some impact on the accuracy of the notes taken. However, we felt it was more important to ensure open and frank dialogue, and the detailed notes were subsequently reviewed by participants to ensure accurate representation. The recommendations in this study are congruent with those proposed by The Ethiopian Ministry of Science and Technology, ${ }^{30} 31$ and also agree with much of the current international literature. This includes the key factors for enabling a research environment as identified by The Health Research System Analysis Initiative of WHO/RPC, ${ }^{21}$ and recommendations for increasing investigator-initiated trial conduct by The European Science Foundation. ${ }^{36}$ Nevertheless it was important to confirm that local investigators in LMICs held similar views as espoused by the above reports. Our subsequent research on this topic has been conducted in other settings, including a wider range of stakeholders in order to overcome these limitations.

\section{CONCLUSION}

Developing research capacity to conduct investigatorinitiated trials is multifaceted and likely to require a different strategy to traditional approaches that focus on individuals or capacity building for specific studies. An appreciation of the barriers and enablers at all levels must be central to development drives. While this study provides a preliminary step forward in this area, further work is needed to test these findings in other settings and to develop the thorough understanding required to successfully support these critical studies.

\section{Author affiliations}

${ }^{1}$ The Global Health Network, Centre for Clinical Vaccinology \& Tropical Medicine, University of Oxford, Oxford, UK

${ }^{2}$ Department of Global Health and Development, London School of Hygiene \& Tropical Medicine, London, UK

${ }^{3}$ Department of Preventive Medicine, College of Health Sciences, Addis Ababa University, Addis Ababa, Ethiopia

${ }^{4}$ Department of Medicine, Faculty of Medicine \& Allied Sciences, Rajarata University of Sri Lanka, Saliyapura, Sri Lanka

${ }^{5}$ Department of Public Health and Hygiene, Faculty of Health Sciences, University of Buea, Buea, Cameroon

${ }^{6}$ Centre for Clinical Vaccinology \& Tropical Medicine, University of Oxford, Oxford, UK

Acknowledgements The authors would like to thank all the faculty, researchers and students who participated in the study in Ethiopia.

Contributors SF conceived, designed and implemented the study, analysed the data and drafted the manuscript with input and assistance from CC, BA and TL. FE, SS and JA collaborated on the interpretation of data and critically revised and reviewed the manuscript. All authors approved the final version of the manuscript.

Funding This work was supported by The Global Health Network, a Bill and Melinda Gates Foundation funded project (grant reference: OPP1053843), and in part by a Nuffield Department of Medicine Doctoral Prize Studentship. The funders had no role in study sponsorship.
Competing interests None.

Ethics approval This study was approved by the University of Oxford Tropical Research Ethics Committee (OXTREC Reference 70-11).

Provenance and peer review Not commissioned; externally peer reviewed.

Data sharing statement No additional data are available.

Open Access This is an Open Access article distributed in accordance with the Creative Commons Attribution Non Commercial (CC BY-NC 3.0) license, which permits others to distribute, remix, adapt, build upon this work noncommercially, and license their derivative works on different terms, provided the original work is properly cited and the use is non-commercial. See: http:// creativecommons.org/licenses/by-nc/3.0/

\section{REFERENCES}

1. Nuyens Y. No development without research. Geneva: Global Forum for Health Research, 2005.

2. Davey S. The 10/90 Report on Health Research 2003-2004. Geneva: Global Forum for Health Research, 2004.

3. Garner P, Kale R, Dickson R, et al. Getting research findings into practice-implementing research findings in developing countries. Br Med J 1998;317:531-5.

4. Lang T, Siribaddana S. Clinical trials have gone global: is this a good thing? PLoS Med 2012;9:e1001228.

5. Page J, Heller RF, Kinlay S, et al. Attitudes of developing world physicians to where medical research is performed and reported. BMC Public Health 2003;3:6.

6. Jones N, Bailey M, Lyytikainen M. Research capacity strengthening in Africa: trends, gaps and opportunities. London: Overseas Development Institute, 2007.

7. Aksoy S. Solutions to neglected tropical diseases require vibrant local scientific communities. PLoS Negl Trop Dis 2010;4:e662.

8. Dayrit MM, Poz MRD, Mercer $\mathrm{H}$, et al. Towards evidence-informed policy-making in human resources for health: the state of research. Global forum update on research for health volume 4. London: Pro-Books Publishing Ltd, 2007:160-2.

9. Devasenapathy N, Singh K, Prabhakaran D. Conduct of clinical trials in developing countries: a perspective. Curr Opin Cardiol 2009;24:295-300

10. Yusuf $S$, Collins R, Peto R. Why do we need some large, simple randomized trials? Stat Med 1984;3:409-22.

11. Godwin M, Ruhland L, Casson I, et al. Pragmatic controlled clinical trials in primary care: the struggle between external and internal validity. BMC Med Res Methodol 2003;3:28.

12. Laabes EP, Desai R, Zawedde SM, et al. How much longer will Africa have to depend on western nations for support of its capacity-building efforts for biomedical research? Trop Med Int Health 2011;16:258-62.

13. Costello A, Zumla A. Moving to research partnerships in developing countries. BMJ 2000;321:827-9.

14. Chandiwana S, Ornbjerg N. Review of North-South and South-South cooperation and conditions necessary to sustain research capability in developing countries. J Health Popul Nutr 2003;21:288-97.

15. Hoekman J, Frenken K, de Zeeuw D, et al. The geographical distribution of leadership in globalized clinical trials. PLOS ONE 2012;7:e45984.

16. Lansang MA, Dennis R. Building capacity in health research in the developing world. Bull World Health Organ 2004;82:764-70.

17. Matsoso $\mathrm{P}$, Auton M, Banoo S, et al. How does the regulatory framework affect incentives for research and development? A proposal for a regulatory framework to improve regulatory capacity and introduce incentives for research and development in areas of public health importance. Geneva: World Health Organisation, 2005.

18. Shafiq N, Pandhi P, Malhotra S. Investigator-initiated pragmatic trials in developing countries-much needed but much ignored. $\mathrm{Br} \mathrm{J}$ Clin Pharmacol 2009;67:141-2.

19. Tyndall A. Why do we need noncommercial, investigator-initiated clinical trials? Nat Clin Pract Rheum 2008;4:354-5.

20. Braun V, Clarke V. Using thematic analysis in psychology. Qual Res Psychol 2006;3:34.

21. World Health Organisation. World report on knowledge for better health: strengthening health systems. Geneva: World Health Organisation, 2004.

22. Gaym A. Health research in Ethiopia-past, present and suggestions on the way forward. Ethiop Med J 2008;46:287-308. 
23. Yassin MA, Datiko DG, Tulloch O, et al. Innovative community-based approaches doubled tuberculosis case notification and improve treatment outcome in Southern Ethiopia. PLOS ONE 2013;8:e63174.

24. Hiatt J. ADKAR: a model for change in business, government and the community. Loveland: Learning Centre Publications, 2006.

25. Whitworth J, Sewankambo NK, Snewin VA. Improving implementation: building research capacity in maternal, neonatal, and child health in Africa. PLoS Med 2010;7:e1000299.

26. Mullan F, Frehywot S, Omaswa F, et al. Medical schools in sub-Saharan Africa. Lancet 2011;377:1113-21.

27. Chen C, Greysen R, Mipando M, et al. SAMSS site visit report: Faculty of Health Sciences, School of Medicine, Jimma University. Ethiopia: SAMMS, 2009. http://www.samss.org/samss.upload/ wysiwyg/Full\%20Site\%20Visit\%20Reports/Jimma\%20-\%20Ethiopia. pdf (accessed 19 Dec 2012)

28. Mbuagbaw L, Thabane L, Ongolo-Zogo $\mathrm{P}$, et al. The challenges and opportunities of conducting a clinical trial in a low resource setting: the case of the Cameroon Mobile Phone SMS (CAMPS) trial, an investigator initiated trial. Trials 2011;12:145.

29. Li LC, Grimshaw JM, Nielsen C, et al. Use of communities of practice in business and health care sectors: a systematic review. Implement Sci 2009;4:27.
30. Ethiopian Science and Technology Agency. National Science, Technology and Innovation (STI) Policy of Ethiopia. Addis Ababa, 2006.http://www.healthresearchweb.org/files/Ethiopia_National_S, T\&I Policy Draft.2006.pdf

31. Ministry of Science and Technology. The Federal Democratic Republic of Ethiopia Science, Technology and Innovation Policy. Addis Ababa, 2012. http://www.most.gov.et/National\%20STI\% 20Policy.htm

32. Datiko DG, Lindtjorn B. Health extension workers improve tuberculosis case detection and treatment success in southern Ethiopia: a community randomized trial. PLoS ONE 2009;4:e5443.

33. Ethiopian Ministry of Health. Policies and Strategies. 2011; 2013(04/ 09/13). http://www.moh.gov.et/English/Information/Pages/Policies\% 20and\%20Strategies.aspx (accessed 04 Sep 2013).

34. Melis T, Ashcroft K, Macfarlane I, et al. Human capacity building needs analysis of Ethiopian new public universities (NPUs). Ethiopia: Development Assistance Group, 2008.

35. Crisp N. Turning the world upside down: the search for global health in the 21st century. London Royal Society of Medicine, 2010.

36. European Science Foundation. Forward LookInvestigator-Driven Clinical Trials. Strasbourg: European Science Foundation, 2009. 